\title{
A New and Easy Technique to Block the Stellate Ganglion
}

\begin{abstract}
Salahadin Abdi, MD, PhD, Yili Zhou, MD,
The stellate ganglion block has been utilized in a variety of painful conditions ranging from sympathetically mediated pain in the upper extremity to the pain associated with intractable angina. A number of techniques are used to block the stellate ganglion.
\end{abstract}

Objective: To describe a new and easy approach to block the stellate ganglion using an oblique view.

Description of a New Technique: The

The technique of stellate ganglion block (SGB) has been ubiquitous among pain specialists for treatment of various painful conditions (1-3). It enjoys utility in sympathetically mediated pain in the upper extremity (2) and atypical facial pain (3) as well as pain associated with intractable angina $(4,5)$. Physicians perform this procedure with or without the aid of fluoroscopy. However, the accuracy of the fluoroscopic approach is much greater than that of a blind approach. A number of techniques are used to block the stellate ganglion. The two methods used most widely to block the stellate ganglion are (1) at the level of the C6 transverse process (Chassaignac's tubercle) without or with the use of using anteroposterior and lateral fluoroscopy and (2) at the level of $\mathrm{C} 7$, the more medial approach at the anterolateral aspect of the

From Department of Anesthesiology, Perioperative Medicine and Pain Management, UM/Jackson Memorial Hospital, Miami, Florida, Advanced Pain Management Center, Milwaukee, Wisconsin, and Neuro Care Network, Tyler, Texas.

Address Correspondence: Salahadin Abdi, MD, PhD, Department of Anesthesiology, Perioperative Medicine, and Pain Management, UM/Jackson Memorial Hospital, 1611 N.W. 12th Ave, C-301, Miami, FL 33136. E-mail: sabdi@med.miami.edu

Funding: There was no external funding in preparation of this manuscript.

Conflict of Interest: None

Acknowledgement: Manuscript received on 5/12/ 04. Revision submitted on 6/30/04. Accepted for publication on $6 / 30 / 04$. patient is placed in the supine position with the new slightly extended, and the head rotated slightly to the side opposite the side to be blocked. The fluoroscopic beam is directed in an anteroposterior direction until the $C_{5} / 6$ disc is well visualized. Subsequently, the fluoroscopic beam is rotated obliquely, ipsilateral to the side where blockade is desired. Under real-time imaging, a single pass is made with a 25-gauge spinal needle to contact the bone. At this point, the nee- dle tip is contacting and resting at the junction between the uncinate process and the vertebral body.

Conclusion: We have described a new approach for stellate ganglion block to be performed under oblique fluoroscopy without technical difficulty or complications.

Keywords: Stellate ganglion block, blind technique, fluoroscopic technique, sympathetic innervation vertebral body as the target $(6,7)$. Even though both techniques may adequately block the stellate ganglion, inadvertent placement of the needle into the vertebral artery, adjacent disc, neural tissue, esophagus, or intrathecal space can have serious consequences.

We describe a new and easy approach to block the stellate ganglion using an oblique view at the level of C7. This approach seems to avoid dangerous structures that can complicate the block.

\section{ANATOMY}

The cervical sympathetic chain is composed of superior, middle, and inferior cervical ganglia. Often, the inferior cervical ganglion is fused with the first thoracic ganglion, forming the stellate ganglion, which commonly measures $2.5 \mathrm{~cm}$

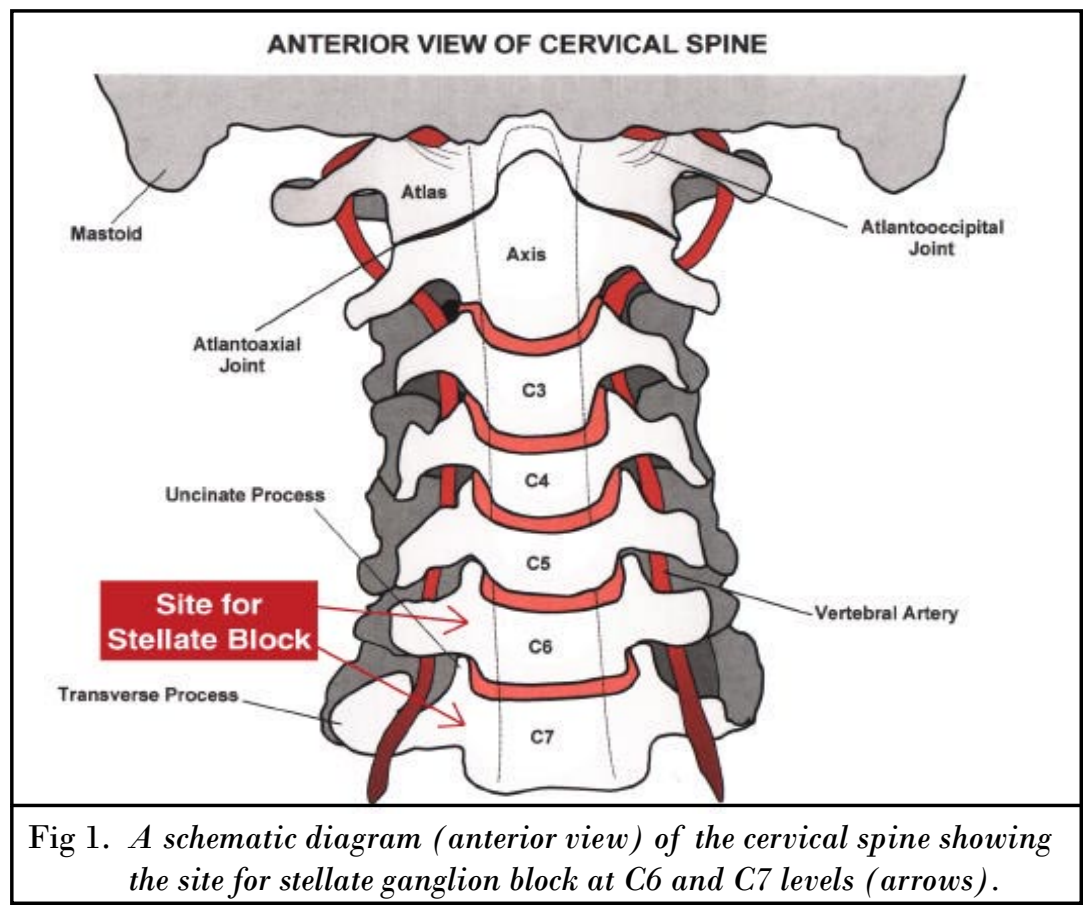




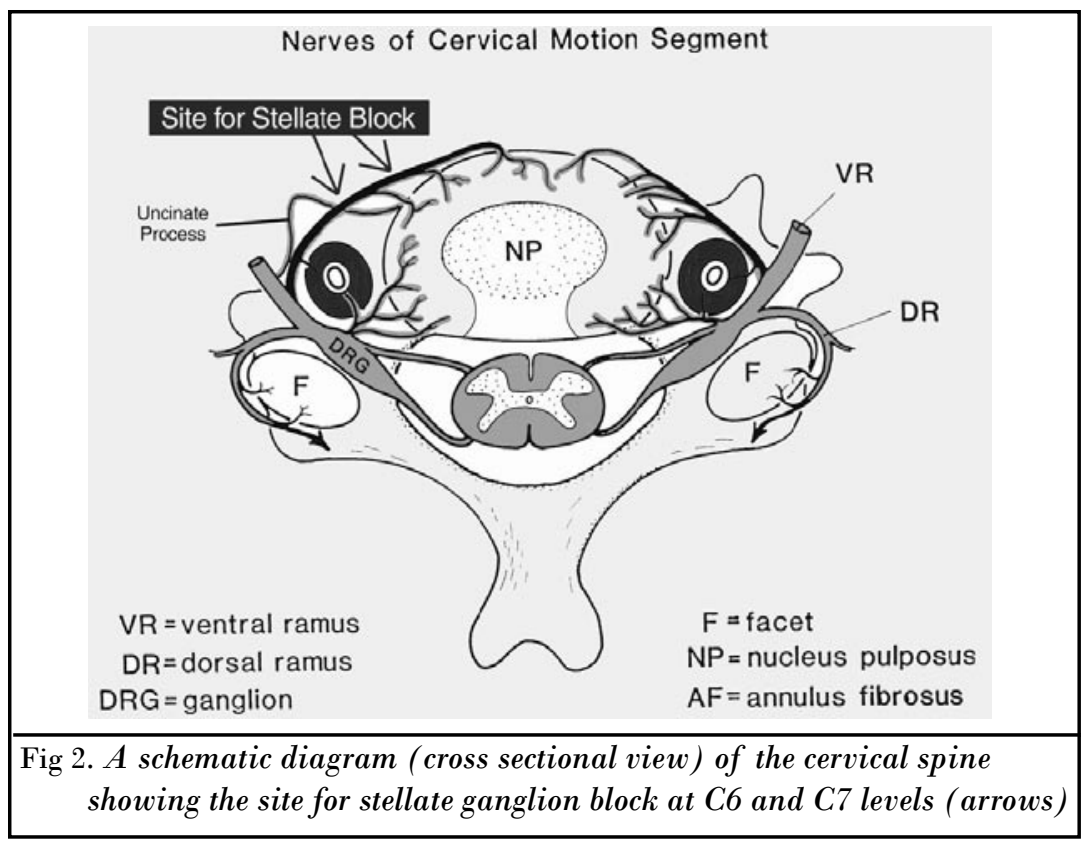

long, $1 \mathrm{~cm}$ wide, and $0.5 \mathrm{~cm}$ thick. It is usually located in front of the neck of the first rib and extends to the interspace between the seventh cervical (C7) and first thoracic (T1) vertebral bodies (2, 8-10). It may rest on the anterior tubercle of $\mathrm{C} 7$ when it is elongated. If the inferior cervical ganglion and first thoracic ganglion are not fused, the inferior cervical ganglion lies in front of the $\mathrm{C} 7$ tubercle, and the first thoracic ganglion rests over the neck of the first rib.

The stellate ganglion lies medial to the scalene muscles, lateral to the longus colli muscle, anterior to the transverse processes and prevertebral fascia, and superior to the subclavian artery and the posterior aspect of the pleura. The initial portion of the vertebral artery lies anterior to the stellate ganglion (Fig. 1).

Preganglionic sympathetic fibers originate from cell bodies in the anterolateral column of the spinal cord. Nerves supplying the head and neck arise from the first and second thoracic spinal segments. Fibers destined to the upper extremities are traceable from the second through the ninth thoracic segments (T2T9). The preganglionic axons leave the $\mathrm{T} 1$ and $\mathrm{T} 2$ ventral roots, pass through the white rami communicans, join the sympathetic chain, and ultimately synapse at the inferior (stellate), middle, or superior cervical ganglion (Fig. 2). Postganglionic sympathetic fibers pass the gray rami and join the cervical or upper cervical plexus. Most of the sympathetic fibers for the head and neck travel along the common and then internal or external carotid artery. Some of the fibers, however, leave the stellate ganglion, form the vertebral plexus, and innervate cranial structures supplied by the vertebral plexus. Thus, blockade of the sympathetic innervations of the head and neck must incorporate inclusion of the stellate ganglion.

\section{INDICATIONS}

The stellate ganglion block has been used to treat vascular insufficiency, vasospasm, hyperhidrosis, and a variety of pain syndromes, including complex regional pain syndrome, type I and II; phantom limb pain; neoplasm; postherpetic neuralgia; herpes zoster; Paget's disease; postradiation neuritis; pain from central nervous system lesions; atypical facial pain and facial neuralgia; and intractable angina pectoris. Most of these indications are based on anecdotal case reports and case series. Well-designed controlled clinical studies are lacking (3). Furthermore, blockade has been found valuable in circulatory problems in the upper extremity such as Raynaud's disease, arterial embolism, accidental intra-arterial injection of drugs, and Ménière's syndrome associated with vascular disease. It has also been indicated as immediate therapy for pulmonary embolus (2).

Absolute contraindications to stellate ganglion blocks include patient refusal, local infection, allergic reaction to local anesthetics, and primary and second- ary coagulopathy. Ideally, stellate ganglion block should be avoided if an affected patient has had a recent cardiac infarction or a severe cardiac conduction block to prevent possible deterioration of cardiac function. Repeated stellate ganglion blocks have been reported to aggravate glaucoma; thus, they are considered as a relative contraindication (2).

\section{DESCRIPTION OF a NeW TECHNIQUE}

Multiple techniques have been previously described $(6,7,11-13)$. The new technique is performed with the patient in the supine position with the neck slightly extended (a pillow may be placed beneath the shoulders), and the head rotated slightly to the opposite side to be blocked. Patients are monitored with electrocardiography, pulse-oximetry, and blood pressure throughout the procedure. The skin temperatures are recorded in the distal portion of both the upper extremities in mirror-image locations. The procedure is performed with a sterile technique.

The fluoroscopy beam is directed in an anteroposterior direction until the C5-C6 disc is well visualized (Fig. 3A, Fig. 4A). This usually requires caudocranial angulations of the $\mathrm{C}$-arm. The $\mathrm{C}$-arm is then rotated obliquely, ipsilateral to the side where blockade is desired. The rotation must occur to allow adequate visualization of the neural foramina (Fig. 3B \& C; Fig. 4B \& C). A skin wheal is raised at the surface point where the junction of the uncinate process and the vertebral body is seen on the fluoroscope (Figs. 14). Under real-time imaging, a single pass is made with a 25 -gauge spinal needle to contact bone at this point. Care should be exercised to avoid passage of the needle toward the neural foramina and the thecal sac, which is exposed posteriorly; the disc located cephalad; and the esophagus, which resides medial to the ultimate target point. In its final position, the needle tip comes to rest at the junction between the uncinate process and the vertebral body (Fig. 4D). The stylet is removed, the extension set is attached, and 1 to $2 \mathrm{ml}$ of radio-opaque contrast is injected to visualize the longus colli muscle (Fig. 4D). The syringe containing the contrast is exchanged with the one that contains the local anesthetic. After ensuring that negative aspiration is performed, a $0.5-\mathrm{mL}$ test dose is injected to rule out intravascular injection into the vertebral artery. The value of this test dose in providing early 

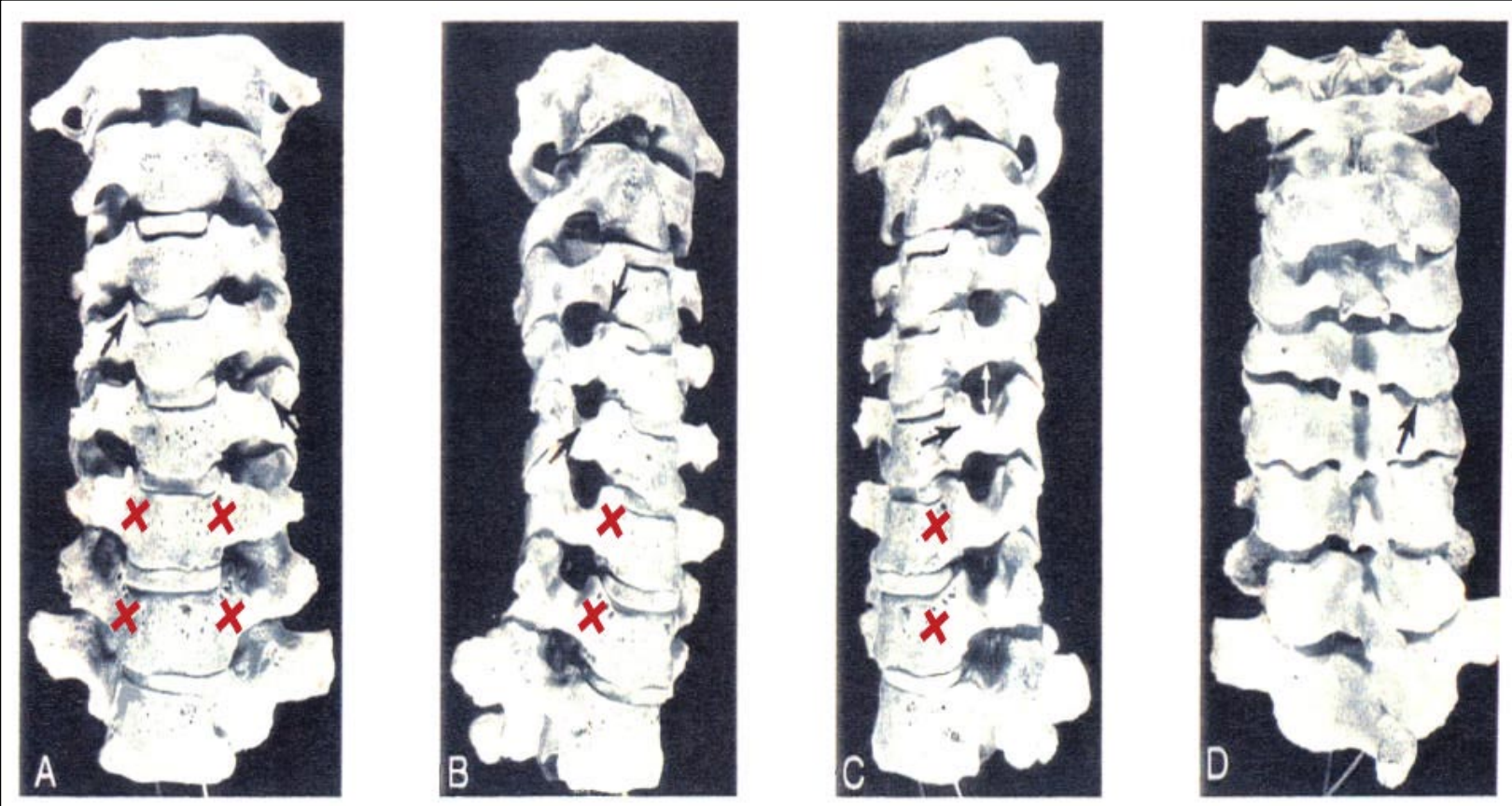

Fig 3. A schematic diagram of the cervical spine in various views, showing the site for stellate ganglion block as marked by " $X "$ "A) anterior view, B) right oblique view, $C$ ) left oblique view, D) posterior view.
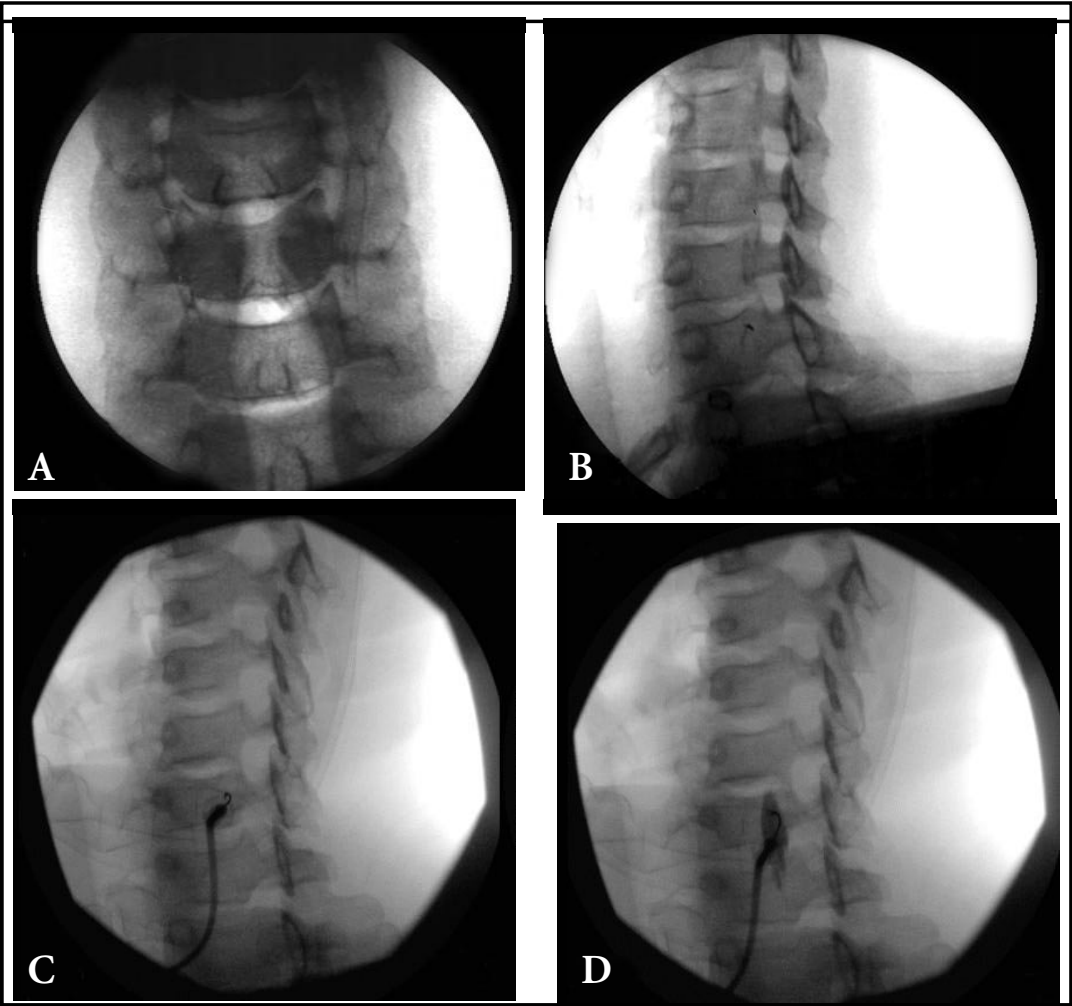

Fig 4. A) The C-arm is in the anterio-posterior position with caudal angulation to optimize disc view with flattened endplates. B) The C-arm is rotated in an oblique manner until the neural foramina are seen. C) Final needle placement at the base of the C7 uncinate process on the left side. D) Stellate Ganglion Block - final position, with contrast injection warning if intra-arterial injection is questionable, as seizures can occur immediately, even with very small volumes of local anesthetic. This is followed by slow injection of 3 to $5 \mathrm{~mL}$ of local anesthetics onto the ganglion. Three to four milliliters of local anesthetic usually is adequate for caudal spread to at least the first thoracic segment.

Meaningful verbal contact should be maintained, and the patient should be urged to respond verbally without moving the head-neck so as to allow recognition of any adverse reaction should it occur. Concomitant hemodynamic monitoring is crucial as well.

If stable in the recovery area for 30 to 60 minutes and tolerating clear liquids without aspiration, the patient is discharged home with an escort. Patients usually develop Horner's syndrome and Guttman's sign, stuffy nose and increased temperature on the ipsilateral side of the block (face and upper extremity) within 5 minutes after the procedure.

We have not encountered any adverse effects thus far with this technique. This method is easily learned and performed by residents and fellows.

\section{DisCUSSION}

Percutaneous stellate ganglion block is widely used to alleviate sympathetical- 
ly maintained upper-extremity pain. Its beneficial effects include elimination or marked reduction in opioids or other pain medication use, increased blood flow, and improved function of those who suffer from sympathetically maintained pain. The prevalent approach is the anterior paratracheal approach with or without fluoroscopy, or with CT imaging or ultrasound guidance to position a needle at C6 or C7 levels. With computed tomography guidance, more precise needle placement and better spread of solution could be ascertained (14).

However, non-fluoroscopic radiologic guidance techniques (MRI, CT, and ultrasound) can be expensive, time-consuming, and impractical for most interventional pain physicians. Another point to consider is the fact that different blocks in the same patient can produce variable spread (12) and may consequently explain the inconsistent outcomes that are seen with a "blind" technique.

Studies using MRI $(9,10)$ or CT (14) have failed to demonstrate any evidence of spread to the stellate ganglion itself, although in the CT study, the stellate ganglion could not actually be visualized. However, an ultrasound imaging study showed spread from C4 to C7 while using $5 \mathrm{ml}$ of local anesthetic and what the authors considered to be an effective clinical stellate ganglion block in 23 of 24 cases (15). Further, Guntamukkala and Hardy (16) performed a cadaver study using methylene blue and observed spread to T4 in most cases.

As regards positioning patients, the supine position for anterior approach offers advantages of more comfort for the patient, avoidance of complications of the prone position, and greater ease in administration of sedatives-analgesic for conscious sedation, if needed, and in managing complications should they arise (convulsions, cardiorespiratory arrest). However, the incidence of other potential complications (pneumothorax; intravascular or intrathecal injection of the local anesthetic) is comparable.

The close proximity of the vertebral artery to the needle used in stellate ganglion block can be hazardous, as injection of local anesthetic into the arterial system can precipitate convulsions. Spread of local anesthetic into the epidural space can result in hypotension and bradycardia besides a risk of subarachnoid injection and total spinal block. Pneumothorax is un- common, but the chance is increased in performing the block at the C7 level. Our review of the CT pleural dome with the oblique technique in non-emphysematous individuals suggests that the pleura is usually avoided and pneumothorax should not be an issue. Associated brachial plexus block is seen in up to $10 \%$ of patients. The recurrent laryngeal nerve lies medially to the sympathetic chain and slightly anterior to it. As local anesthetic spreads, this may also be blocked, resulting in a hoarse voice and a unilateral vocal cord paresis. The probability of this occurring has been demonstrated to be $10 \%$ after the use of $10 \mathrm{ml}$ of local anesthetic, rising to $80 \%$ when $20 \mathrm{ml}$ of bupivacaine was used (17). The development of a Horner's syndrome is usually taken as being demonstrative of an accurate stellate ganglion block (18). This is not normally troublesome, although some case reports have cited its occurrence bilaterally (19), which obviously may cause visual impairment. Conversely, Horner's syndrome does not ensure blockade of the upper-extremity sympathetic nervous system, as the latter arises from the T1/T2 segments.

Our new technique for the stellate ganglion block was developed to overcome these technical difficulties and to improve the safety and efficacy of the block. We performed the stellate ganglion blocks at the base of the uncinate process of the C7 vertebrae using oblique fluoroscopy. Careful placement of the needle in close proximity to the ganglia increases the efficiency of the sympathectomy, reduces the volume of the local anesthetic agent required, and diminishes the risk of complications. In this study, the correct needle position was confirmed radiographically, and the injected contrast solution was shown to fill the same vertebrotransverse junction plane as seen in the classic block. Thus, 3 to $5 \mathrm{ml}$ was enough to achieve a favorable clinical effect in our patients.

Malmqvist et al (18) reported that the Chassaignac tubercle is not suitable for placement of the tip of the needle during a cervical sympathetic block, because this may result in inadequate distribution of the local anesthetic and thus inadequate sympathetic block of the upper extremity. Furthermore, an arterial puncture and subsequent seizure may occur. Thus, introducing the needle at the base of the uncinate process of the C7 vertebrae increases the likelihood of encoun- tering the cervical sympathetic ganglia. By its very nature, the blind technique requires large volumes that will have aberrant spread (including contralaterally), and this in part may explain some of the adverse effects $(20,21)$ associated with the classic blind method. Methylene blue injection with the classical blind approach at C6 was thoracoscopically visualized at the stellate ganglia in only $46 \%$, while with the same blind technique at $\mathrm{C} 7$ the dye was seen at the stellate ganglion in $63 \%$ of cases (22).

The recorded and unrecorded case logs of complications from the classic method have driven sagacious interventionists to advocate image-guided cervicothoracic blockade (23). Indeed, such an approach has demonstrated increased clinical effectiveness of fluoroscopically guided injection as compared to blind injection (24). In the description by Elias (23), a paratracheal anterior approach (fluoroscopic) guided by palpation of the C6 tubercle was made in a perpendicular manner until the $\mathrm{C} 6$ or $\mathrm{C} 7$ tubercle was contacted. This may lead to paresthesia, as the nerve root may be encountered.

Furthermore, the anterior paratracheal (fluoroscopic) approach does not ensure inadvertent insertion into the thecal sac, the esophagus, the disc, or the neural tissue, as these structures cannot be adequately defined with the straight anteroposterior fluoroscopic view. If this approach must be used, we suggest flattening the end plates and visualizing the disc. A bony endpoint is then chosen, and the needle placement is guided.

This advice is based on observing the technique of numerous prominent physicians (from academic and private-practice arenas and during cadaver courses) who have inserted needles into the disc, the thecal sac, and the nerve root using the straight anteroposterior paratracheal approach. The intent is not to banish the fluoroscopic anterior paratracheal approach - it is important for safety and effectiveness over the pervasive classic blind injection-but rather to elucidate potential pitfalls that are not readily appreciated or emphasized.

Conversely, the advantages of our new technique are as follows:

- Eliminates pressing or pushing the vascular system out of the way

- Eliminates pressing on the Chassaignac tubercle, which can be uncomfortable and even painful to 
patients

- Minimizes the chance of intravascular injection

- Minimizes the chance of esophageal perforation

- Minimizes the chance of recurrent laryngeal nerve paralysis

- Reduces the volume of local anesthetics needed to cover lower cervical through upper thoracic areas

- Most important, can easily be learned by trainees

The experience obtained in the present technique suggests that in addition to efficacy, the technique is safe but also easily learned. However, even the new technique that we have defined is not the panacea as, theoretically, in the individual with emphysematous bullous pleura, an oblique C7 insertion to the base of the uncinate process can lead to a pneumothorax. Our approach is more medial than other image-guided approaches described in the literature and, though this should serve to offset the possibility of pleural puncture, it also results in local anesthetic delivery marginally distant from the true location of the stellate ganglion. Regardless of the promises inherent in our approach, prospective literature validating its effectiveness is a prerequisite to widespread adoption in the clinical setting.

\section{ConcLusion}

A blind technique or the paratracheal anterior approach using high volume may or may not adequately block the stellate ganglion but often leads to blockade of adjacent nerve structures as well, with possible complications. We have successfully used our new approach under oblique fluoroscopy view for stellate ganglion blocks in our patients without technical difficulty or complications.

\section{ReFERENCES}

1. Bonica JJ. The Management of Pain. Philadelphia, Lea \& Febiger, 1953.

2. Raj PP. Stellate ganglion block. In Waldman and Wenner (eds). Interventional Pain Management. Philadelphia, Saunders, 1996.

\section{Author Affiliation: \\ Salahadin Abdi, MD, PhD \\ Chief of Pain Medicine \\ Professor of Anesthesiology \\ UM/Jackson Memorial Hospital \\ 1611 N.W. 12th Ave. C-301 \\ Miami FL 33136 \\ E-mail: sabdi@med.miami.edu}

\section{Yili Zhou, MD, PhD}

Jackson Memorial Hospital, Dept. of

Anesthesiology

1611 NW 12th Ave.

Miami FL 33136

E-mail: yz019@yahoo.com

\section{Nilesh Patel, MD}

Advanced Pain Management Center

Milwaukee, WI

5900 S. Lake Drive

Cudahy, WI 53110

\section{Bhupinder Saini, MD}

Advanced Pain Management Center

5900 S. Lake Drive

Cudahy, WI 53110

E-mail: BSSaini@aol.com

\section{John Nelson, MD}

Pain Care Centers of America

1625 Medical Center Point, Suite 240

Colorado Spring CO 80907

E-mail: johnnelsonmd@yahoo.com

3. Rauck R. Sympathetic nerve blocks: Head, neck and trunk. In Raj PP (ed). Practical Management of Pain, 3rd ed. Philadelphia, Mosby, 2000, pp 651-682.

4. Wiener L, Cox JW. Influence of stellate ganglion blockade on angina pectoris and the post exercise electrocardiogram. Am J Med Sci 1966; 252:289-295.

5. Hammond C, Leach AA, Chester MR. Temporary sympathectomy in refractory angina. Heart 1999; 81(suppl):56.

6. Moore DC, Bridenbaugh, LD Jr. The anterior approach to the stellate ganglion. JAMA 1956; 160:158-162.

7. Moore DC. Anterior (paratracheal) approach for block of the stellate ganglion. In Moore DC (ed). Regional Block. Springfield, IL, Charles C. Thomas, 1981, 123137.
8. Ellis H, Feldman S. Anatomy for Anesthetists, 3rd ed. Blackwell Scientific Publications, Oxford, 1979, pp 256-262.

9. Hogan QH, Erickson SJ, Haddox JD, Abram $\mathrm{SE}$. The spread of solutions during stellate ganglion block. Reg Anesth 1992; 17:7883.

10. Hogan QH, Erickson SJ. MR imaging of the stellate ganglion: Normal appearance. $A m$ J Radiat 1992; 158:655-659.

11. Bryce-Smith R. Stellate ganglion block. Anaesthesia 1952; 7:154-156.

12. Davies RM. Stellate ganglion block-a new approach. Anaesthesia 1952; 7:151153.

13. Carron H, Litwiller R. Stellate ganglion block. Anesth Analg 1975; 54:567-570.

14. Christie JM, Martinez CR. Computerized axial tomography to define the distribution of solution after stellate ganglion nerve block. I Clin Anaesth 1995; 7:30611.

15. Kapral S, Krafft P, Gosch M et al. Ultrasound imaging for stellate ganglion block: Direct visualization of puncture site and local anesthetic spread. Reg Anesth 1995; 20:323-328.

16. Guntamukkala M, Hardy PAJ. Spread of injectant after stellate ganglion block in man: An anatomical study. Br J Anaesth 1991; 66:643-644.

17. Hardy PAJ, Wells JCD. Extent of sympathetic blockade after stellate ganglion block with bupivacaine. Pain 1989; 36:193-196.

18. Malmqvist EL, Bengtsson M, SorensenJ. Efficacy of stellate ganglion block: A clinical study with bupivacaine. Reg Anesth 1992; 17:340-347.

19. Wallace MS, Milholland AV. Contralateral spread of local anesthetic with stellate ganglion block. Reg Anesth 1993; 18:5559 .

20. Allen G, Samson B. Contralateral Horner's syndrome following stellate ganglion block. Can Anaesth Soc J 1986; 33:112113.

21. Leong MS, Mackey S. Delayed subdural block after a stellate ganglion block. Anesthesiology 2001; 94:358-359.

22. Harano K et al. Difference of spread of injected solutions after stellate ganglion block to the sixth and seventh vertebral levels. Anesthesiology 1998; 89:1159.

23. Elias M: Cervical sympathetic and stellate ganglion blocks. Pain Physician 3 :294304, 2000.

24. Hsu ES, et al. Repeat stellate ganglion block under fluoroscopic guidance on postherpetic neuralgia. Reg Anesth Pain Med 26:93 9, 2001. 
